\title{
09 | El viaje de Jorge Oteiza a la Exposición Universal de Bruselas de 1958: de la crítica al proyecto arquitectónico_Emma López-Bahut
}

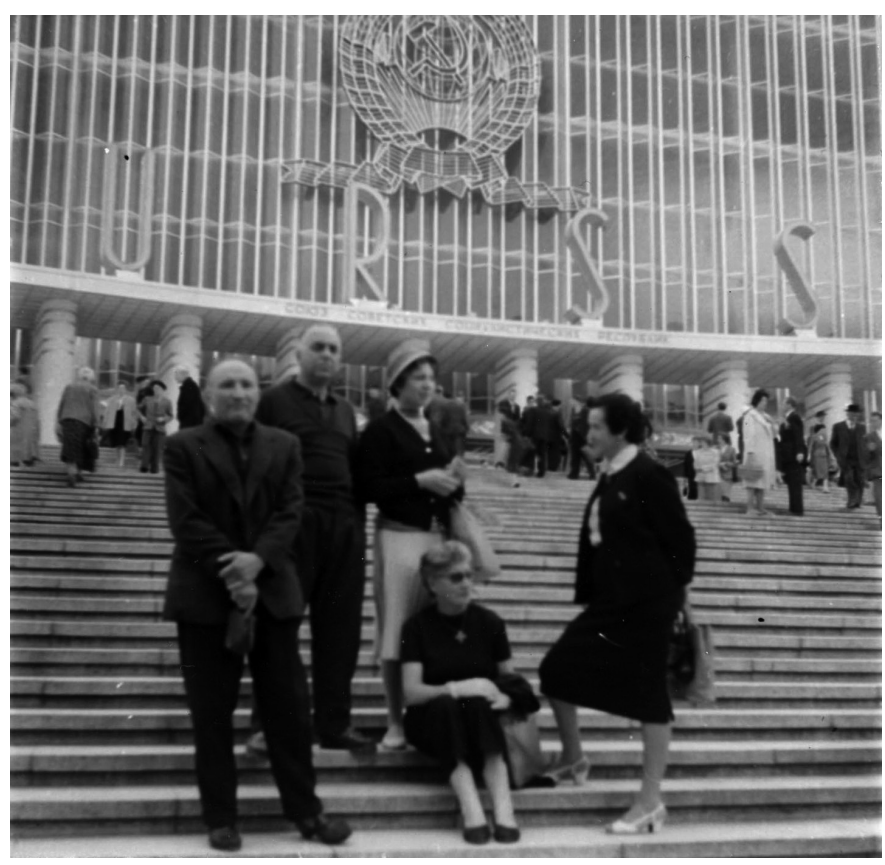

[1]

"Yo vengo de aquí, que es como estar fuera de todo, casi como no estar. Vengo de España a visitar unos países donde se trabaja en un clima de libertad creadora... También vengo de juzgar. Siempre he juzgado con benevolencia porque trataba de enseñar, de enseñarme a mí." ${ }^{1}$ Jorge Oteiza

El viaje constituye una herramienta fundamental para la construcción de la mirada arquitectónica. La experiencia del cuerpo, el habitar completo del espacio y del tiempo, es insustituible por cualquier otro conocimiento (construcciones virtuales, fotografías, planos, teorías, etc.). El viaje en sí mismo, el trayecto, el camino que se recorre, es tan importante como el objetivo final. Un recorrido, a veces inesperado, que, evocando experiencias, percepciones, recuerdos, sensaciones, etc., se conecta con un modo de proyectar, de imaginar la arquitectura ${ }^{2}$. Frente a lo virtual que nos inunda, la apuesta por la experiencia y su recuerdo, por el espacio vivido ${ }^{3}$.

Para los arquitectos españoles de los años cincuenta el viaje al extranjero constituyó un aprendizaje primordial para el desarrollo de la arquitectura en un país congelado tras la Guerra Civil y el periodo autárquico de los cuarenta. Esta generación de arquitectos también se ayudó de colaboraciones con artistas que, en un proceso similar, intentaban recuperar el tiempo perdido logrando alcanzar, en ambos casos, una presencia internacional a finales de la década ${ }^{4}$. La integración de las artes era una cuestión que se debatía a nivel internacional y también en España, tal como expresaba Alberto Sartoris en su conferencia en Madrid en 1958: "el problema que urge resolver es, desde luego, el de la integración de las artes en la arquitectura"5.

En esa frontera difusa de intercambios y aprendizajes de arquitectos y artistas durante los cincuenta, el escultor Jorge Oteiza fue un personaje clave pues, al mismo tiempo que desarrollaba su escultura, estaba inmerso en el ambiente arquitectónico no solo como mero observador o colaborador, sino como activo creador 6 . Obtener el premio internacional al mejor escultor en la IV Bienal de Arte de São Paulo en 1957, supuso un punto de inflexión en su trayectoria escultórica ${ }^{7}$. Pero también en la arquitectura: reflexiones sobre la integración de las artes y la arquitectura; trabajos en fachadas de edificios como en el Hotel Fénix en Madrid o Backook en Bilbao ${ }^{8}$; y propuestas arquitectónicas como el Memorial al Padre Donosti o el Monumento a José Batlle en Montevideo.

En 1958 Oteiza viajó a la Exposición Universal e Internacional de Bruselas, en la que había participado elaborando, junto con un equipo multidisciplinar, el montaje interior del Pabellón español, obra de J.A. Corrales y R. Vázquez Molezún, que fue premiado con la Medalla de Oro de la
Resumen pág 57 | Bibliografía pág 62

Emma López-Bahut (Burgos, 1974). Doctora Arquitecta por la Universidade da Coruña (2013). Máster en Diseño Arquitectónico por la Universidad de Navarra (2004). Arquitecta por la ETSA de Madrid (2002). Desde 2009, profesora de Proyectos arquitectónicos en la ETSA da Coruña. Becas de investigación por la Fundación Museo Jorge Oteiza (2005-2007) y por el Ministerio de Exteriores MAE-AECID (2008). Investigación sobre las colaboraciones entre arquitectos $y$ artistas españoles, que ha expuesto en artículos, congresos y seminarios internacionales, publicando De la escultura a la ciudad. Monumento a Batlle en Montevideo. Oteiza y Puig, 1958-60 (Pamplona, 2007) y colaborando en la edición crítica del libro de Jorge Oteiza Ejercicios Espirituales en un túnel (Pamplona, 2011).

\section{Palabras clave}

Jorge Oteiza, Bruselas, París, Exposición Universal, Expo'58, Pietilä, Schwanzer, arquitectura, escultura, espacio

\footnotetext{
1 OTEIZA, J. El viaje es una estatua [escrito, 1 hoja]. Madrid: 23 de abril 1954. Alzuza (Navarra): Archivo de Jorge Oteiza, Fundación Museo Jorge Oteiza, ID: 3220. Disponible en: http://www.museooteiza.org/catalogos/documentacion/detalles.php?id=3220\&bd=fondod

2 Cf. MORENO MANSILLA, L. Apuntes de viaje al interior del tiempo. Barcelona: Fundación Caja de Arquitectos, 2001, p. 13.

3 "El espacio arquitectónico es espacio vivido más que espacio fisico, y el espacio vivido siempre trasciende la geometría y la mensurabilidad". PALLASMAA, J. Los ojos de la piel. La arquitectura y los sentidos, Barcelona: Gustavo Gili, 2006, p. 64.

${ }^{4}$ Cf. RUIZ CABRERO, G.; MOLINS P. (eds.). L'arquitectura i l'art dels anys 50 a Madrid. Barcelona: Fundación La Caixa, 1996; PÉREZ, C. (dir.). España años 50. Una década de creación. Madrid: Sociedad Estatal para la Acción Cultural Exterior, 2004.

5 SARTORIS, A. "Perspectivas acerca de la integración de las artes en la arquitectura". Revista de Ideas Estéticas. 1958, diciembre. p. 265. Conferencia celebrada en Madrid el 24 de junio de 1958, dentro de la I Conferencia de Arquitectura y Vivienda, organizada por Fomento de las artes, y recogida posteriormente en el artículo señalado.

${ }^{6}$ Cf. LÓPEZ-BAHUT, E., "Oteiza y lo arquitectónico. De la estatua-masa al espacio urbano (1948-1960)" Directores: José Juan González-Cebrián Tello; Carlos Labarta Aizpún. Universidade da Coruña, Escuela Técnica Superior de Arquitectura, Departamento de Proyectos Arquitectónicos y Urbanismo, 2013. 7 Cf. BADIOLA, T. "Oteiza Propósito experimental". En: Oteiza: Propósito Experimental. Madrid: Fundación Caja de Pensiones, 1988, p. 29; ÁLVAREZ, S. Jorge Oteiza. Pasión y razón. San Sebastián: Nerea, 2003, pp. 28-30.
} 
${ }^{8}$ Cf. LÓPEZ-BAHUT, E. "El muro como trabajo espacial: los relieves de Jorge Oteiza en la arquitectura (1951-1958)". VLC arquitectura. Universitat Politècnica de València. 2014, vol. 1, no 1 , pp. 31-58.

${ }^{9}$ Cf. FEDUCHI, P. Archipiélago hexagonal. En CÁNOVAS, A. (ed). Pabellón de Bruselas '58. Corrales y Molezún. Madrid: Ministerio de Vivienda, 2005, pp. 103-120.

10 Documentación conservada en el Archivo de Jorge Oteiza en la Fundación Museo Jorge Oteiza en Alzuza (Navarra), desde ahora con la sigla AFMJO y con su número de registro ID. Aunque en este artículo se hace referencia a los documentos tal como han sido catalogados, estos escritos forman parte de un conjunto documental que Oteiza elaboró para preparar la conferencia "La ciudad como obra de arte", celebrada en Valencia el 11 de noviembre de ese año. Cf. LÓPEZ-BAHUT,

E. "La ciudad como obra de arte. Origen de la reflexión espacial de Jorge Oteiza sobre la ciudad". En: Oteiza y la crisis de la modernidad. Actas del Primer Congreso Internacional Jorge Oteiza. Pamplona: octubre de 2010. pp. 415-423.

11 Se ha podido identificar a los compañeros de viaje de Oteiza gracias a la referencia recogida en el periódico local: "Marcharon a Francia y Bélgica los señores de Oteiza (don Jorge), Montes Iturrioz (don Manuel) e Ibargoyen (don Miguel)". El Bidasoa, Irún, 1958, junio, nº 672, sin paginar.

12 En AFMJO: Pabellón de la URSS en Expos'58 ID: 2465 y 6114; Hotel en Bruselas ID: 2808 y 6109; Catedral de Tours ID: 2831 y 6111; Catedral de Chartres, ID: 6110.

13 La referencia a la catedral de Reims se recoge en: OTEIZA, J. [Sobre arquitectura y el Gótico] [escrito, 1 hoja]. 1958. AFMJO,

ID: 8164. Disponible en: http://www.museooteiza.org/catalogos/documentacion/detalles. php?id=8164\&bd=fondod. La referencia a Verdún se recoge en: OTEIZA, J. [Sobre mi escultura y proyecto de estela funeraria] [escrito y dibujos, 1 hoja]. 1958. AFMJO, ID: 8163. Disponible en: http://www.museooteiza.org/catalogos/documentacion/detalles. php?id=8163\&bd=fondod

14 OTEIZA, J. [Sobre arquitectura y el Gótico], cit.

15 Ibídem.

16 Ibídem.

17 Cf. OTEIZA, J. "Para un entendimiento del espacio religioso. El crómlech-estatua vasco y su revelación para el arte contemporáneo". El Bidasoa, Irún, 1959, $n^{\circ} 724$, sin paginar.

18 Oteiza anotaba en el manuscrito del artículo: "Este espacio religioso, planteado no desde lo religioso sino desde lo estético, y desde lo estético no como expresión sino como receptividad pura, sería el espacio religioso común a todas las construcciones en el arte religioso". OTEIZA, J. Para un entendimiento del espacio religioso en la arquitectura y en el arte [escrito, 4 hojas], junio 1959. AFMJO, ID: 7995, p. 4. Disponible en: http://www.museooteiza.org/ catalogos/documentacion/resultado.php?b$\mathrm{d}=$ fondod. También en su libro Quousque Tandem...! aparece la misma imagen de la catedral de Tours, explicando "El espacio religioso (estéticamente como vacio-cromlech) en el gótico de Tours, suspendido en lo alto con el empuje exterior de los arbotantes". OTEIZA, J. Quousque Tandem...! Ensayo de interpretación estética del alma vasca. San Sebastián: Auñamendi, 1963, pp. 55-58.
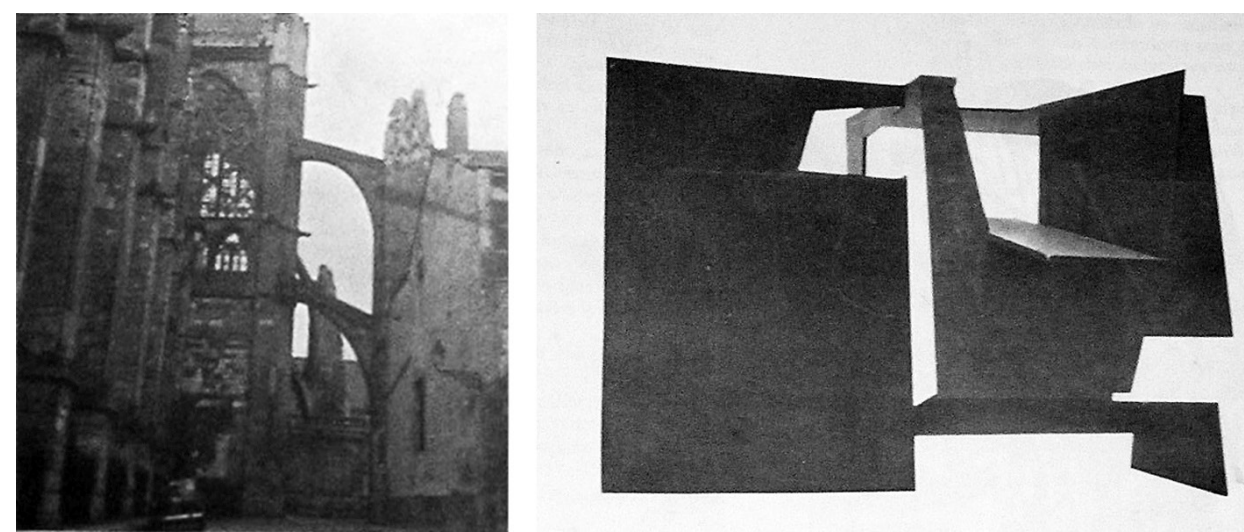

[2]

muestra9 ${ }^{9}$ Partiendo del material fotográfico y de los escritos originales conservados en su archivo personal, se ha reconstruido el viaje que Oteiza realizó desde Irún hasta Bruselas, con el objetivo de conocer los ejemplos arquitectónicos concretos que visitón ${ }^{10}$. A partir de estos, se profundiza en las siguientes tres cuestiones: primera, sus reflexiones sobre el espacio sagrado, que le llevan a establecer paralelismos entre la catedrales góticas y sus piezas escultóricas; en segundo lugar, el modo de integración del arte y la arquitectura que Oteiza defiende y cuál rechaza; y, por último, se establece la influencia directa en el desarrollo del Monumento a Batlle de dos pabellones de la Expo'58, el Pabellón de Austria, obra de Karl Schwanzer y Gran Premio de Arquitectura de la muestra, y el Pabellón de Finlandia, obra de Reima Pietilä. De este modo se constata la conexión de Oteiza con la arquitectura desde los procesos creativos que, en este caso como en otros anteriores, parten de cuestiones y referencias arquitectónicas.

\section{El viaje hasta Bruselas (Irún-Tours-Chartres-París-Verdún-Bruselas)}

En junio de 1958 Oteiza emprende su viaje desde su residencia en Irún (País Vasco) hasta Bruselas, acompañado por su esposa, Itziar Carreño, y de los iruneses Manuel Montes Iturrioz y Miguel lbargoyen, con sus respectivas esposas ${ }^{11}$. Las fotografías conservadas en su archivo han permitido establecer parte del recorrido del viaje ${ }^{12}$. Partieron desde Irún en coche y, antes de llegar a París, realizaron al menos dos paradas en Francia: en Tours y Chartres. En las anotaciones de Oteiza aparecen referencias de Reims y de Verdún ${ }^{13}$. Ya en Bruselas, visitaron la Exposición Universal, fotografiándose frente al Pabellón de la U.R.S.S. [1].

Gracias a la visita a las catedrales góticas francesas de Tours, Reims y Chartres, Oteiza establece un paralelismo de estas arquitecturas con las estatuas que estaba realizando pues considera que todos son "objetos espaciales absolutos"14. Escultura y arquitectura son contenedores de espacio, que considera sagrado porque está destinado a acoger espiritualmente a las personas. Señala especialmente la catedral de Tours debido a que su estructura, mucho más aligerada que la de Chartres, se traslada y se separa en el exterior obteniendo "la caja del espacio interior sagrado y luminoso en el aire"15. Y advierte que en su escultura se invierte la relación que se da en estos templos góticos: "mi estatua es arquitectura (relación del gótico invertido. Finalidad espiritual semejante y formalismo inverso)"16. Esta conexión la expone en su artículo "Para un entendimiento del espacio religioso. El crómlech-estatua vasco y su revelación para el arte contemporáneo"17, relacionando la fotografía exterior de la catedral de Tours con su pieza Homenaje a Mallarmé [2]. El espacio religioso, tanto en escultura como en arquitectura, es un espacio desocupado, libre de elementos formales, que obtiene su valor sagrado desde lo estético ${ }^{18}$.

Aunque no se conservan fotografías de su estancia en París, Oteiza señala la sede de la Unesco en la capital francesa como una mala solución de integración del arte con la arquitectura y con la ciudad. Obra de los arquitectos Marcel Breuer, Pier Luigi Nervi y Bernard Zehrfuss (1958), nace con la idea de conseguir una obra de arte integral a partir de la intervención de seis artistas: Picasso, Moore, Arp, Calder, Miró y Noguchi. Oteiza critica que se ha intervenido desde una belleza entendida como decoración, una colocación posterior de obras artísticas en la arquitectura, no habiéndose producido un verdadero trabajo colaborativo entre artistas y arquitectos ${ }^{19}$. Quizá, para ilustrarlo, Oteiza realiza un collage superponiendo algunas de sus Cajas Vacías sobre el material gráfico de la sede de la Unesco que se recoge en la revista Aujourd' hui: art et architecture ${ }^{20}$ [3].

\section{En la Expo'58}

Desde la historiografía la Exposición Universal de Bruselas no ha sido considerada como un acontecimiento arquitectónico clave, aunque se señalan como obras relevantes el Pabellón Philips de 
Le Cobusier y el alemán de Eiermann y Ruf ${ }^{21}$. La muestra potenciaba la recuperación de la confianza en una tecnología que, como había quedado patente en la Segunda Guerra Mundial, era capaz de arrasar el mundo. Por ello, la arquitectura se caracterizó por alardes ingenieriles en la mayoría de los edificios, bautizándose la arquitectura como "festival de estructuras"22, constituyendo un buen ejemplo el pabellón-icono de la muestra, el Atomium. Coincidiendo con algunas de las críticas de sus contemporáneos, como las de Rogers ${ }^{23}$, Oteiza consideraba que esta apuesta por la tecnología era totalmente errónea, pues si la arquitectura "se apoya en la técnica de construir, la arquitectura se expresa como ingeniería (pabellón francés de la Exposición de Bruselas)"24. Señala un pabellón que fue, junto con el de Estados Unidos y el belga Marie Thumas, una exhibición estructural de las posibilidades de las grandes cubiertas colgantes ${ }^{25}$.

Revisando las filmaciones de la exposición, especialmente las nocturnas, se hace patente que el movimiento, el color, la luz y el sonido eran parte fundamental del ambiente de la Expo'58 ${ }^{26}$. Oteiza considera que se trata de un camino equivocado y lo identifica también en situaciones que se dan en las ciudades actuales, como el estímulo visual de la publicidad:

"La reacción contra ese tipo falso de agitación artística se ha iniciado desde un terreno precisamente colectivo y no por el artista: la lucha contra el ruido de la ciudad y en la cita internacional de la Exposición de Bruselas. Y la reacción del ciudadano contra el alboroto de los medios de reproducción de discos mezclados y sin interrupción con voces, avisos, noticias, contra el falso sistema continuo de ocupación del espectador. Contra la supresión o asesinato del silencio" ${ }^{27}$.

El edificio más destacado en la Expo'58 fue el Pabellón Philips, realizado por Le Corbusier con la colaboración de Edgar Varèse en la música, Jean Petit y Philippe Agostini en el espectáculo visual y la participación de lannis Xenakis como músico y arquitecto. Se planteaba una interacción total entre la música electrónica, el montaje visual y la propia arquitectura. Para ello se creó el "Poème électronique", que lograba que edificio, música, palabra e imágenes proyectadas conformasen una propuesta de arte total. Extrañamente Oteiza en sus reflexiones no hace referencia directa a este pabellón, y más aún habiéndose mostrado interesado por el trabajo de Le Corbusier ${ }^{28}$. Sin embargo, condena el aumento de expresión basada en el movimiento y la luz, tanto en el arte como en la arquitectura, señalando algunos artistas y arquitectos:

"El arte actual, incomprensiblemente orientado en la misma línea de la naturaleza y del progreso mecánico pretende superar aquellos estímulos concentrándonos en construcciones y montajes enriquecidos por la dinámica formalista de los colores, el sonido, la luz y el movimiento. La conducta visible del artista contemporáneo, identificada con el pensamiento de artistas y arquitectos -Moholy-Nagy, Giedion, Calder, Sert, etc.- y que se acepta en las publicaciones y en los coloquios más importantes sobre el arte como creación independiente o en combinación con la arquitectura" ${ }^{29}$.

[3]

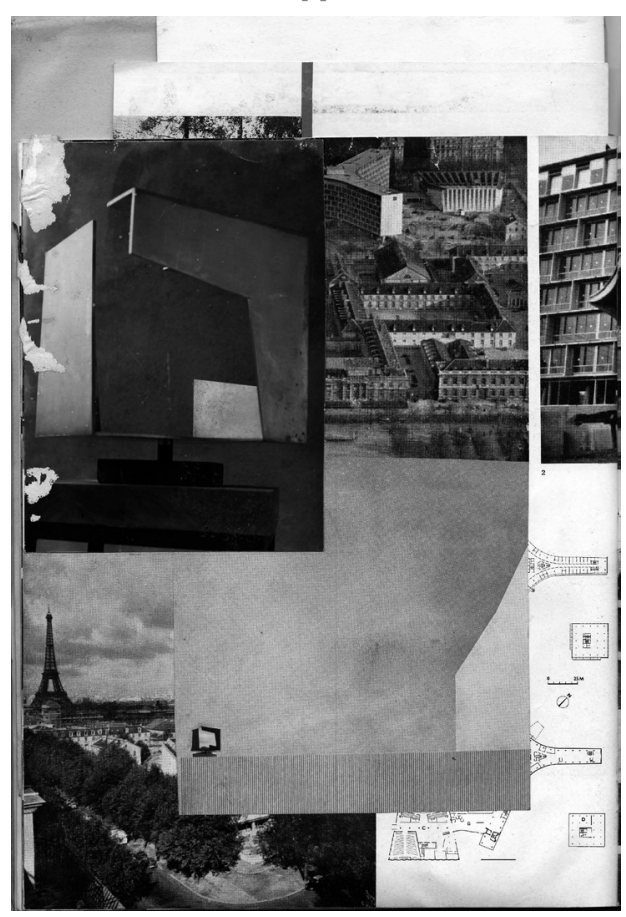

19 Oteiza señalaba sobre la sede de la Unesco en Paris: "... el panorama actual del arte tiene que parecerme pura superficial solución decorativa en lo que respecta a la obra de arte en función del hombre, en cuanto a los problemas de integración del arte con la ciudad no hemos pasado por alto el planteamiento secundario de los problemas de la belleza al pobre estilo tradicional repetido en la Unesco". OTEIZA, J. La escultura contemporánea se ha detenido [escrito, 4 hojas]. Septiembre de 1958. AFMJO, ID: 18806, p. 3. Disponible en: http://www. museooteiza.org/catalogos/documentacion/ detalles.php?id=18806\&bd=fondod

20 "Le siècle de I'UNESCO. Paris". Aujourd'hui: art et architecture. Boulogne (France): septiembre 1958, $\mathrm{n}^{\circ} 18$, pp. 64-74. Ejemplar conservado en el AFMJO.

${ }^{21}$ Cf. DE KOONING, M.; DEVOS, R. La arquitectura de la Expo'58. En: En CÁNOVAS, A. (ed). Pabellón de Bruselas '58. Corrales y Molezún. Madrid: Ministerio de Vivienda, 2005, p. 67.

22 "Architecture at Brussels: Festival of Structures". Architecture Records, junio $1958, n^{\circ} 6$. ${ }^{23}$ Rogers afirmó, además de criticar el gigantismo de algunos pabellones como los de la URSS y USA, que: "L'errore non è solo nella valutazione degli effetti, ma nell'impostazione concettuale dell'opera, nella sua assoluta inaderenza al mondo dell'arte" [El error no solo está en la evaluación de los efectos, sino en la creación de la obra conceptual, en su absoluto desapego del mundo del arte]. ROGERS, E. N. "A l'Expo '58 il futuro (dell'architettura) non è cominciato". Casabella Continuità. Milán: $1958, n^{\circ} 221$, pp. 2-21

24 OTEIZA, J. Desviaciones actuales de la arquitectura y el arte: sus causas y su corrección [escrito, 4 hojas]. ca. 1958. AFMJO, ID: 10077, p. 1. Disponible en: http://www. museooteiza.org/catalogos/documentacion/ detalles. php?id=10077\&bd=fondod

25 Cf. DEVOS, R.; ESPION, B. "The hanging roofs of Expo 58: knowledge, prestige and ideals". En: Proceedings of the ICE - Engineering History and Heritage. 2012, vol. 165, n 3, pp.187-196.

${ }^{26}$ Videos sobre la Exposición Universal de Bruselas disponibles en: http://www.youtube. $\mathrm{com} /$ watch $? v=\mathrm{PaU} 8 \mathrm{Cn} \mid Z$ WCA\&feature $=$ pla yer_embedded (5' 13"- 6' 39"). http://www. youtube.com/watch? $\mathrm{v}=\mathrm{TfbdE}-4 \mathrm{z} 8 \mathrm{Jw}$ ( 6 ' 24"- 6' 59") [consultados el 27 de diciembre de 2013]. Cf. DEVOS, R. Lights for dazzle and structure : the lighting of streets, squares, buildings and exhibits at Expo 58. En: L'architecture lumineuse au XXe siècle = Luminous architecture in the 20th century. Gante (Bélgica): Snoeck, 2012, pp. 15-25.

27 OTEIZA, J. Para Naturaleza de lo abstracto [escrito, 4 hojas]. ca. 1958. AFMJO, ID: 8228, p. 4. Disponible en: http://www.museooteiza org/catalogos/documentacion/detalles.php?i$\mathrm{d}=8228 \& \mathrm{bd}=$ fondod\#prettyPhoto.

${ }^{28}$ La iglesia de Ronchamp de Le Corbusier es la única obra arquitectónica que aparece como referencia en: OTEIZA, J. Propósito experimental 1956-1957 [catálogo]. Madrid, 1957.

29 OTEIZA, J.; PUIG, R. [Memoria del proyecto de monumento a Batlle] [escrito, 2 hojas]. 1958. AFMJO, ID: 18054, pp. 7-8. Disponible en: http://www.museooteiza.org/catalogos/ documentacion/detalles. php?id=18054\&b$\mathrm{d}=$ fondod.

${ }^{30}$ OTEIZA, J.; PUIG, R., [Memoria del proyecto de monumento a Batlle], cit., p. 7.

31 OTEIZA, J. Integración del arte en la arquitectura [escrito, 3 hojas]. ca. 1958. AFMJO, ID: 8008, p. 1. Disponible en: http://www. museooteiza.org/catalogos/documentacion/ detalles. php?id=8008\&bd=fondod 
El Pabellón Philips parece estar alejado de lo que Oteiza considera una auténtica colaboración del arte y la arquitectura, señalando otros pabellones como ejemplo.

\section{El pabellón de Finlandia: integración del arte y la arquitectura}

Uno de los ejes centrales del pensamiento de Oteiza en 1958 es la integración del arte y la arquitectura, cuyas conclusiones materializa en el Monumento a José Batlle, distinguiendo diferentes grados de relación entre ambas disciplinas. El nivel más bajo de integración sucede cuando la obra de arte se instala en el espacio arquitectónico, con el fin de dar servicio espiritual al hombre que lo habita. Conserva su autonomía pero debe ser definida desde el espacio arquitectónico en el que se ubica ya que, de este modo, evita ser un agregado decorativo. Por tanto, Oteiza se aleja de una postura más radical que promovía la desaparición total de la obra de arte, defendida por Mondrian y por el grupo De Stijl:

"Para Mondrian, la integración del arte con la arquitectura suponía una colaboración orientada a la construcción exclusiva del espacio de la arquitectura como creación espiritual, sin obra de arte. Para nosotros, en una etapa complementaria y final, se recupera la obra de arte desde ese espacio espiritual, pero con un arte igual a cero (cero como expresión formal)"30.

La integración máxima propuesta por Oteiza consiste en que la pieza artística garantice estéticamente ese espacio arquitectónico, llegando a un punto en que como tal (pintura, mural, estatua, fotografía, etc.) sea innecesaria gracias a un intenso trabajo de colaboración entre el artista y el arquitecto. Como ejemplo, Oteiza considera la arquitectura de Alvar Aalto:

"Alvar Aalto trabaja con este concepto de integración. Y si ondula un muro o presiona o abre un elemento de la arquitectura, es como si trabajase con artistas que intervendrían de este modo, para hacer del espacio habitable una construcción espiritual" ${ }^{31}$.

El muro ondulante al que hace referencia se identifica con el interior del Pabellón de Finlandia en la Exposición Universal de Nueva York (1939), en el que el espacio se articulaba mediante un triple muro curvo, escalonado e inclinado, con un claro carácter escultórico. Oteiza no pone la atención en el muro, sino en el espacio habitable que genera, afirmando que una verdadera integración se produce cuando el arquitecto trabaja con el espacio del mismo modo que él en su escultura: un espacio destinado para recibir al habitante, material y espiritualmente, evitando generar un espectáculo formal. Una construcción espiritual desde lo estético, cuya materia es el espacio, como identifica en su visita a la catedral de Tours.

En el mismo sentido que el pabellón de Aalto, Oteiza considera el Pabellón de Finlandia de la Expo'58 un ejemplo de integración de arte y arquitectura:

"En la muestra de arquitectura de Bruselas: el pabellón de Finlandia, resuelto y veraz en sí mismo frente al pabellón alemán, aparentemente nuevo, hecho para el espectador. La riqueza espacial

[3] Jorge Oteiza, Collage realizado en varias hoias del articulo sobre la nueva sede de la UNESCO en Pars publicado en Aujurdhu la art et architecture (1958). Fuente: "Le siècle de I'UNESCO. Paris". Aujourd'hui: art et architecture. Boulogne (France): septiembre 1958, $n^{\circ}$ 18, p.p 64-74. Ejemplar conservado en el Archivo Fundación Museo Jorge Oteiza.

[4] Egon Eiermann y Sep Ruf, Pabellón de Alemania en la Exposición Universal de Bruselas, Bélgica, 1958. Fuente: "Pavillon de l'Allemagne occidentale". L'architecture d'aujourd'hui. Boulogne (France): junio 1958 $n^{\circ} 78$, p. 16.
[4]

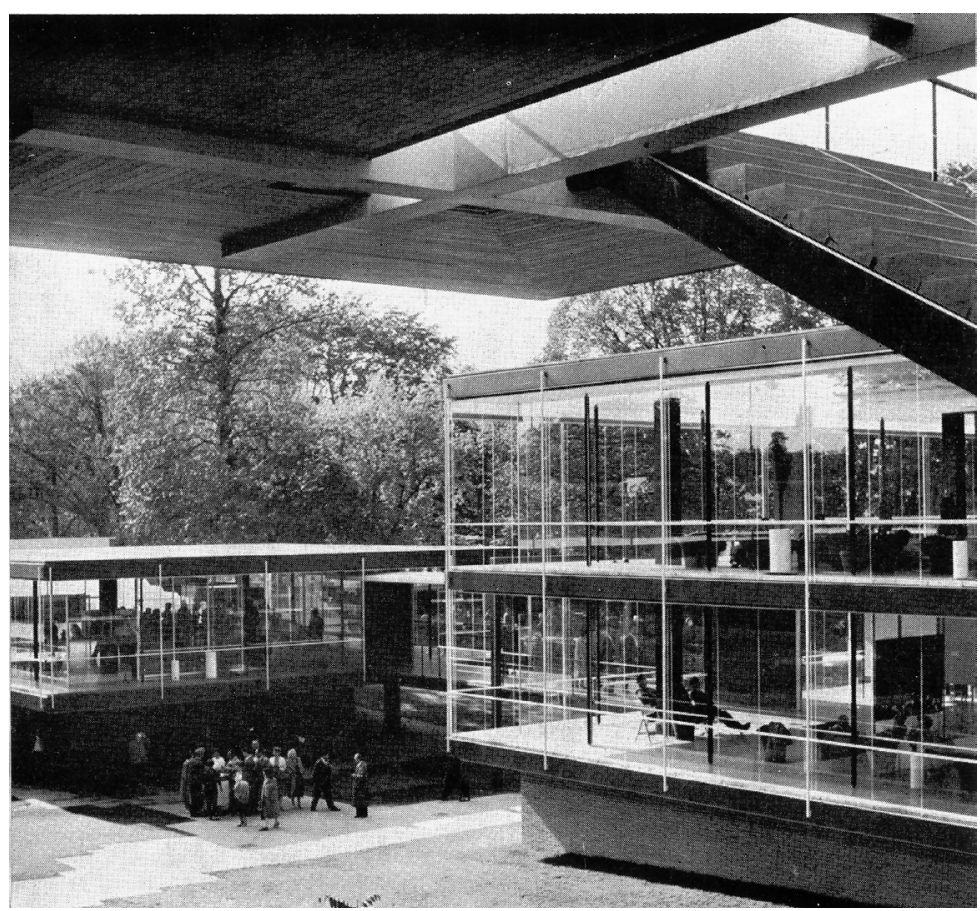


de Finlandia frente a la pesadez íntima, al material crudo y muerto de Alemania. Actúa en sí mismo o actúa para el espectador" ${ }^{32}$.

El pabellón alemán [4], obra de Egon Eiermann y Sep Ruf, era un edificio absolutamente transparente, elevado, que se organizaba en ocho piezas conectadas entre sí mediante galerías abier$\operatorname{tas}^{33}$. Oteiza entiende que la desmaterialización del objeto arquitectónico basada en la transparencia supone que el habitante obtiene infinitos puntos de vista. El edificio, al ser transparente no genera un espacio contenido, sino que expande este espacio hacia todas las direcciones, incorporando lo exterior, el movimiento, el entorno. El espacio se diluye y el tiempo cobra presencia. La naturaleza se incorpora al pabellón rompiendo la idea de espacio abstracto, vacío y desocupado de formas que Oteiza defendía, tanto en sus estatuas como en el Monumento a Batlle.

Al contrario que el pabellón alemán, el de Pietilä se vuelca a su interior sin perder por ello fuerza y carácter en el exterior, con un ritmo muy marcado por el módulo que varía en altura, más bajo en la zona central, donde se produce la entrada, creciendo hacia los dos laterales [5]. El edificio es un gran espacio expositivo continuo, al que se accede desde el punto central, con un cuerpo más bajo adosado de servicio y cafetería. El material empleado es la madera, tanto en el interior como en fachada. Una vez dentro, el espacio crece trapezoidalmente en planta y se expande en altura por el módulo que rige el edificio [6]. El plano del falso techo cubre todo el interior y se inclina de manera uniforme en una sola dirección. Es realizado con listones de madera separados entre sí que permiten tamizar la luz que se introduce desde los lucernarios de la cubierta, aunque finalmente se pintaron para lograr que la luz natural no llegase a penetrarse en él. El diseño del interior fue realizado íntegramente por el artista finlandés Tapio Wirkkala, como indica Pietilä:

"Del complejo original, formado por el pabellón y por la exposición, que yo he proyectado, no he tomado parte más que en el exterior, haciendo el encargo el gobierno finlandés al artista Tapio Virkala [sic] para organizar la exposición"34.

A pesar de no existir colaboración entre Pietilä y Wirkkala, Oteiza encuentra en este pabellón un ejemplo de síntesis de la escultura y pintura con la arquitectura, pues no aparecen colocadas o superpuestas a la arquitectura, sino que desde ella han sido definidas. Y el pabellón, como sucedía en el de Aalto, posee cualidades estéticas gracias a su trabajo desde el espacio:
32 OTEIZA, J. [Notas sobre estética] [escrito 5 hojas]. ca. 1958. AFMJO, ID: 8223, p.3. Disponible en: http://www.museooteiza.org/ catalogos/documentacion/detalles.php?i$\mathrm{d}=8223 \&$ bd $=$ fondod\#prettyPhoto

33 Cr. BOYKEN, I. Egon Eiermann / Sep Ruf: Deutsche Pavillons, Brüssel 1958. Stuttgart; Londres: Edition Axel Menges, 2007.

34 "Del complesso originale, formato dal padiglione e dall'esposizione, che io ho progettato, non ho avuto parte che negli esterni, avendo incaricato il Governo finlandese l'artista Tapio Virkala [sic] di organizzare l'esposizione". PIETILÄ, R. "Il padiglione finlandese". Casabella Continuità, Milán, 1958, n 221, p. 22.

35 OTEIZA, J. Desviaciones actuales..., cit., p. 3.

${ }^{36}$ Estas referencias, tanto al cementerio de Verdún como al Pabellón de Austria, Oteiza las estaba planteando al mismo tiempo que desarrollaba el proyecto para Monumento a Batlle que enviarian al concurso a finales de diciembre de 1958 y se encuentran entre las notas de preparación de la conferencia "La ciudad como obra de arte" que impartió en Valencia el 11 de noviembre. Por tanto, estas referencias se producen en un estado de ideación del proyecto arquitectónico, todavia sin concluir.

${ }^{37}$ Después de la Exposición de Bruselas el pabellón fue trasladado a Viena, convirtiéndose en el Museo de Arte Moderno y, entre 1962 y 2002, se conformó como Museo de Siglo XX, cf. HOFMANN, W. "Das Museum des 20. Jahrhunderts in Wien". Werk, junio 1965, n52, pp. 222-227. Posteriormente fue reformado de la mano del discipulo de Schwanzer, Adolf Krischanitz, para convertirse, en 2011, en la 21er Haus. Cf. Sin autor, "History of the 21er Haus", en 21er Haus, Austria, Disponible en: http://www.21erhaus. at/en/21er-haus/geschichte-des-21er-haus [consultado 09/02/2014].

[5]
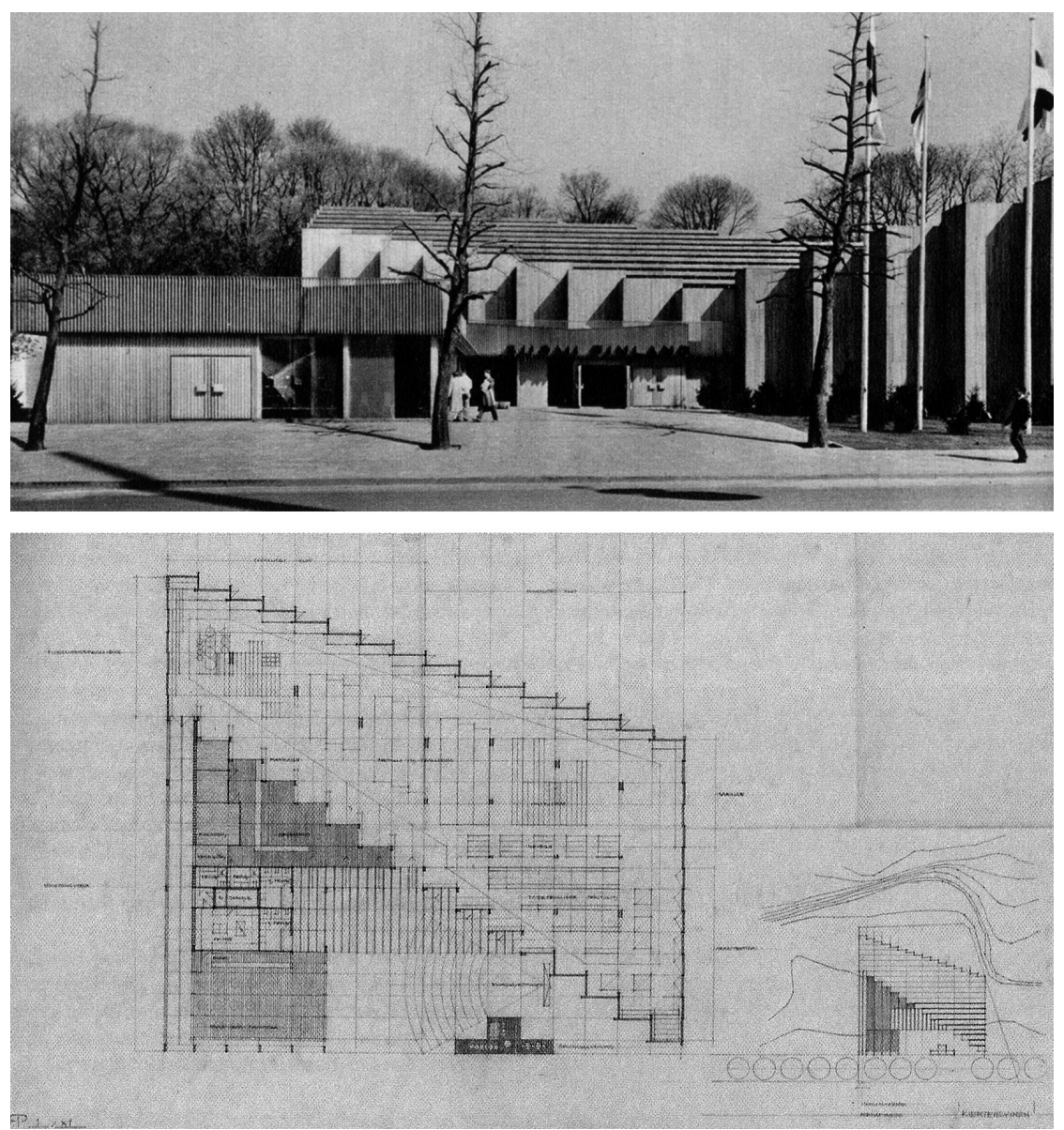

[5] Reima Pietilä, Pabellón de Finlandia en la Exposición Universal de Bruselas, Bélgica, 1958; vista exterior y planta general. Fuente: Arriba: "Prime immagini di Bruxelles". Domus. Milán: agosto $1958, n^{\circ} 345$, p. 14 . Abajo: $R$ aili: Reima Pietilä. Un desafío a la arquitectura moderna [Catálogo]. Madrid: Fundación ICO; Museum of Finnish Architectura; Johansson E., 2009 , p. 89.

[6] Reima Pietilä, Pabellón de Finlandia en la Exposición Universal de Bruselas, Bélgica, 1958; montaje expositivo del diseñador finlandés Tapio Wirkkala. Fuente: ROGERS, E. N. “Al'Expo'58 il futuro (dell'architettura) non è cominciato". Casabella Continuità. Milán: $1958, n^{\circ} 221$, p. 20 
“... Suficiente el E [espacio] como cálculo racional dentro de la casa y fuera en la ciudad si el E [espacio] es atendido como disposición final para el espíritu. Aquí la integración del arte con la arquitectura, no muestra la presencia práctica del pintor ni del escultor. El tratamiento del espacio asume la pronunciación plástica no visible y ortográficamente, sino con acento oculto o tónico. Esta me parece ser la interpretación de las ideas de PM [Piet Mondrian]. El pabellón de finlandés de [sic] en Bruselas constituía en este sentido la más libre prodigiosa de las creaciones actuales. Pintura y escultura terminan en la arquitec. [arquitectura]. El edificio se convierte en arqui [arquitectura], la ciudad en obra de arte independientes del trabajo visible de pintor y esc. [escultor]"35.

\section{El pabellón de Austria y el cementerio de Verdún como referencias arquitectónicas}

Entre París y Bruselas se encuentra el cementerio de Douaumon, que Oteiza toma como referencia para configurar el Monumento a Batlle. Situado en la localidad francesa de Verdún, es un cementerio-monumento a las víctimas de la batalla que tuvo lugar allí en la Primera Guerra Mundial. Consta de un gran edificio-osario con un imponente elemento vertical, a cuyos pies se sitúan las tumbas de los soldados caídos. A través de dos pequeños croquis [7], Oteiza explica el proceso de síntesis formal de los elementos de este cementerio para conformar los del proyecto del Monumento a Batlle [8]. Su intención es trasladar la idea de sagrado y de monumentalidad que identifica en Verdún a su propuesta para Montevideo ${ }^{36}$.

En ese mismo escrito, Oteiza señala el pabellón de Austria que, habiendo constatado su viaje a la Expo'58, se identifica con el construido por el arquitecto vienés Karl Schwanzer y que obtuvo el Gran Premio de Arquitectura de la muestra ${ }^{37}$. Se trata de un edificio conformado por tres cuerpos independientes, dos rectangulares a ras de suelo y el principal elevado que parece flotar a seis metros [9] gracias a cuatro potentes pilares de hormigón sobre los que cargan cuatro grandes vigas de las que se suspende toda la estructura metálica [10]. De este modo, la planta baja es un espacio cubierto y abierto. En el cuerpo elevado se genera un gran vacío central, definido en torno a los cuatro pilares, sobre el que vuelca todo el edificio, pues el cuerpo elevado hacia el exterior es opaco. En uno de los laterales aparecen unas escaleras metálicas que conectan la zona de exposición elevada con el plano del suelo. Los materiales empleados son acero y vidrio. El interior es sobrio, coherente con el carácter de la arquitectura [11].

¿Por qué Oteiza toma como referencia este pabellón para el proyecto del Monumento a Batlle? Se trata de un edificio que se aleja de los alardes estructurales de muchos pabellones de la Expo'58

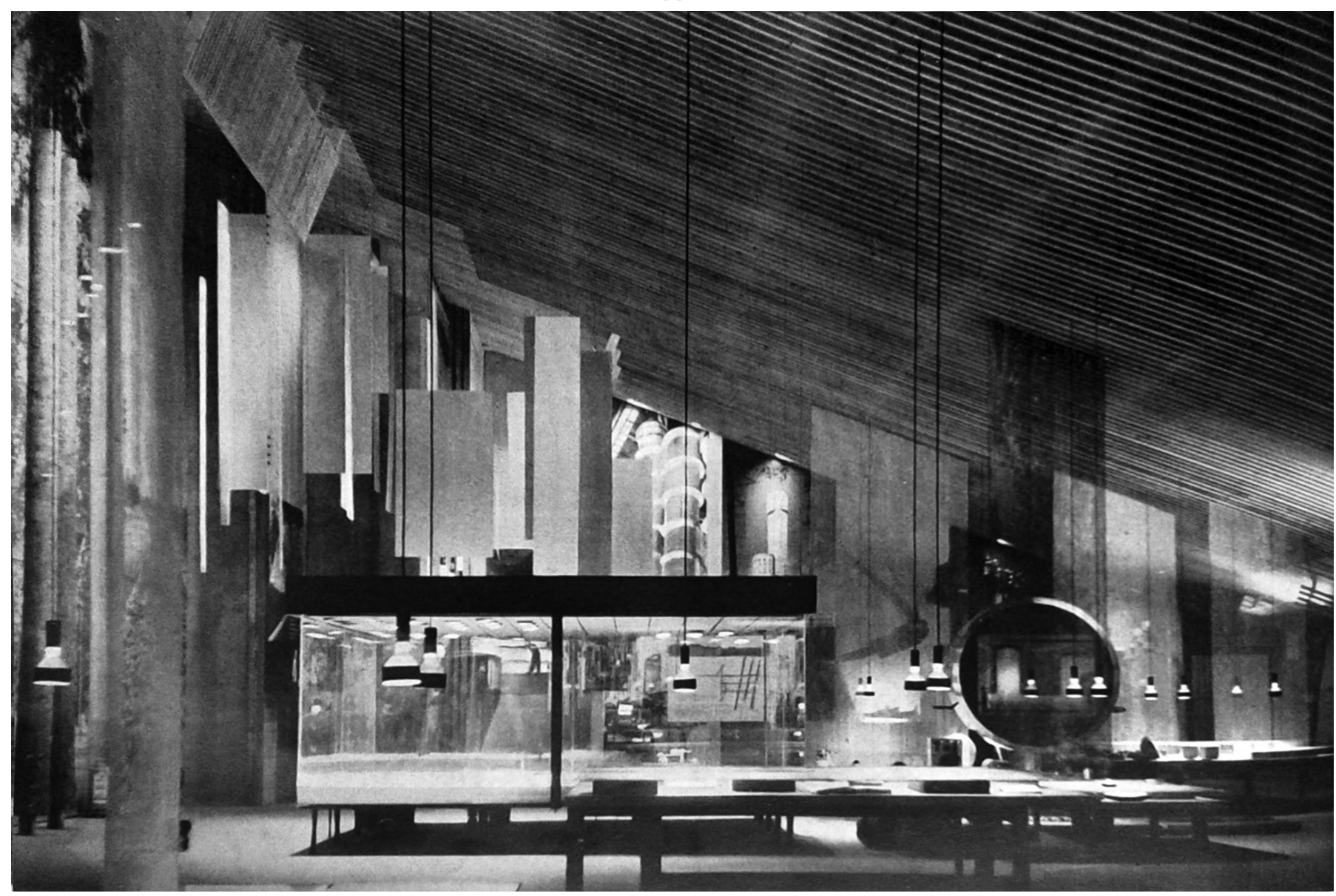




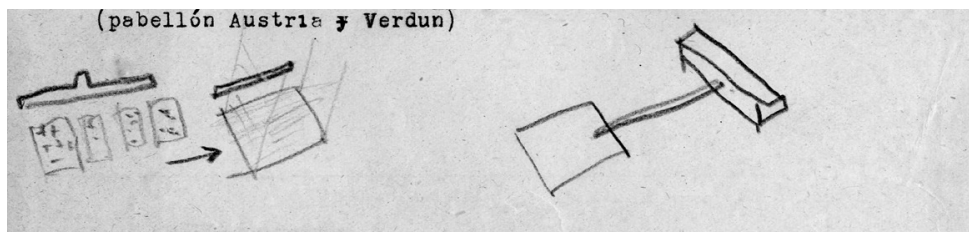

[7]

pues la estructura está presente pero de manera esencial, mínima, y al servicio de la arquitectura. Se puede definir como una gran pieza blanca, elevada, cerrada al exterior y, sin embargo, luminosa, que genera un gran acceso cubierto donde aparece la estructura sustentante del edificio. La zona expositiva es un espacio contenido, controlado, aislado y diseñado íntegramente. Frente al pabellón alemán totalmente abierto al exterior y partícipe de la naturaleza, el pabellón de Austria se cierra en sí mismo. Las formas terminan pesando menos que el espacio contenido. La temática del pabellón se centra en la cultura artística austriaca, haciendo hincapié en la música, y se expone en el interior con gran sobriedad, donde apenas diferenciamos arquitectura y montaje expositivo.

Existen claras semejanzas entre la propuesta de edificio de Puig y Oteiza y el pabellón austriaco de Schwanzer [12]:

-Se separan del suelo gracias a una estructura que no tiene un gran protagonismo, generando una planta baja cubierta pero abierta que sirve de acceso, que en el caso del Monumento a Batlle, es un espacio más limpio, más vacío. En el pabellón aparecen dos piezas prismáticas de menos altura que alojan usos como un auditorio o la cafetería.

-Se cierran en sí mismos, pues sus fachadas no se abren a lo que les rodea. En el pabellón sus paramentos verticales exteriores son opacos y los interiores se abren a un patio central que organiza el conjunto. En el Monumento a Batlle también todos los cierres verticales de la pieza elevada son opacos y, mediante un sistema de lucernarios continuo, la pieza se abre hacia el cielo.

-La estructura secundaria está presente. En el pabellón marca el ritmo de fachada de la zona elevada mientras que en el monumento la estructura es visible al interior generando así una imagen más abstracta del edificio

Oteiza y Puig parecen tomar los aspectos del Pabellón de Austria y llevarlos a un punto más extremo en el Monumento a Batlle: la disposición de la pieza elevada es más clara, el edificio es más abstracto y contundente, su estructura tiene menos presencia y el espacio interior está más volcado hacia sí mismo pues solo se abre hacia el cielo.

\section{Conclusiones}

Se pueden establecer referencias formales del Monumento a Batlle con arquitecturas conocidas por Oteiza en alguno de sus viajes anteriores, como el Pabellón Ciccilio Matarazzo que acogió la IV Bienal de São Paulo (1957), obra de Oscar Niemeyer y H. Uchôa; o de arquitectos que le interesaban como el Museo de la Paz en Hiroshima (1955) de Kenzo Tange, al que Oteiza homenajeó en una sus piezas; o semejanzas que establece él mismo, como el Museo Conmemorativo de la fundación de la ciudad de Brasilia (1959), obra de Niemeyer ${ }^{38}$.

Sin embargo, tal como demuestran sus reflexiones, son los edificios que conoce en su viaje de Irún a Bruselas, el habitar imprescindible para comprender la arquitectura, lo que le permite tomar una posición crítica sobre cuestiones arquitectónicas de su tiempo. Se sirve de esta experiencia para avalar sus reflexiones sobre el modo de integrar arquitectura y arte, recogidas en su conferencia "La ciudad como obra de arte" (Valencia, 1958). Además, es capaz de establecer la relación entre las catedrales góticas como contenedores de espacio y sus esculturas, desde el concepto de espacio religioso, definido como aquel que por sus cualidades es capaz de trascender y desligado de una determinada religión. De un modo similar al que Le Corbusier plantea al hablar del "espacio inefable":
[7] Jorge Oteiza, dibujos explicativos sobre el Monumento a Batlle y el cementerio de Verdún, junto a la referencia del pabellón de Austria. Fuente: OTEIZA, J. [Sobre mi escultura y proyecto de estela funeraria] [escrito y dibujos, 1 hoja]. 1958. Alzuza (Navarra): Archivo de Jorge Oteiza, Fundación Museo Jorge Oteiza, ID: 8163. Disponible en: http://www. museooteiza.org/catalogos/documentacion/ detalles.php? id=8163\&bd=fondod.

[8] Jorge Oteiza y Roberto Puig, Monumento a José Batlle, Montevideo, Uruguay, 1958 (primera fase concurso); vista suroeste. En primer plano, frente a la colina, el cuadrado negro. Flotando sobre él, la gran viga volada que surge desde debajo del edificio. Fuente: Alzuza (Navarra): Archivo de Jorge Oteiza, Fundación Museo Jorge Oteiza, ID: 19257.

[9] Karl Schwanzer, Pabellón de Austria en la Exposición Universal de Bruselas, Bélgica 1958; vista exterior. Fuente: "All'Exposizionie Universale di Bruxelles". Domus. Milán: noviembre $1958, n^{\circ} 347$, p. 1

[10] Karl Schwanzer, Pabellón de Austria en la Exposición Universal de Bruselas, Bélgica, 1958; vista exterior nocturna. Fuente: "Pavillon de l'Austrie". L'architecture d'aujourd'hui. Boulogne (France): junio $1958, n^{\circ} 78$, p. 18.

[11] Karl Schwanzer, Pabellón de Austria en la Exposición Universal de Bruselas, Bélgica, 1958; interior de la zona de exposición. Fuene: "All'Exposizionie Universale di Bruxelles". Domus. Milán: noviembre 1958, n 347 , p. 2.

[12] Comparación del pabellón de Austria y el Monumento a Batlle. Arriba: Karl Schwanzer, Pabellón de Austria en la Exposición Universal de Bruselas, Bélgica, 1958; sección por el patio interior de la pieza elevada. Abajo: Jorge Oteiza, Roberto Puig, Monumento a José Batlle en Montevideo, Uruguay, 1958; alzado. Fuentes: Arribe: "Pavillon de l'Austre". LarchiFuentes: Ariba: "Pavilon de 'Austie'. L'archi1958, n 78, p. 18. Abajo: OTEIZA, J.; PUIG, R. Concurso de monumento a José Batlle en Montevideo. Arquitectura. Madrid: 1959, $\mathrm{n}^{\circ}$ 6 , p. 23 


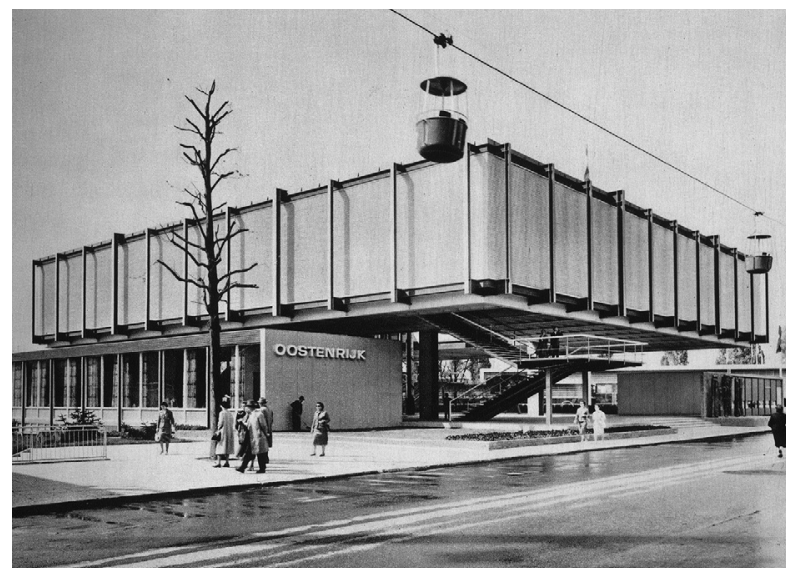

[9]

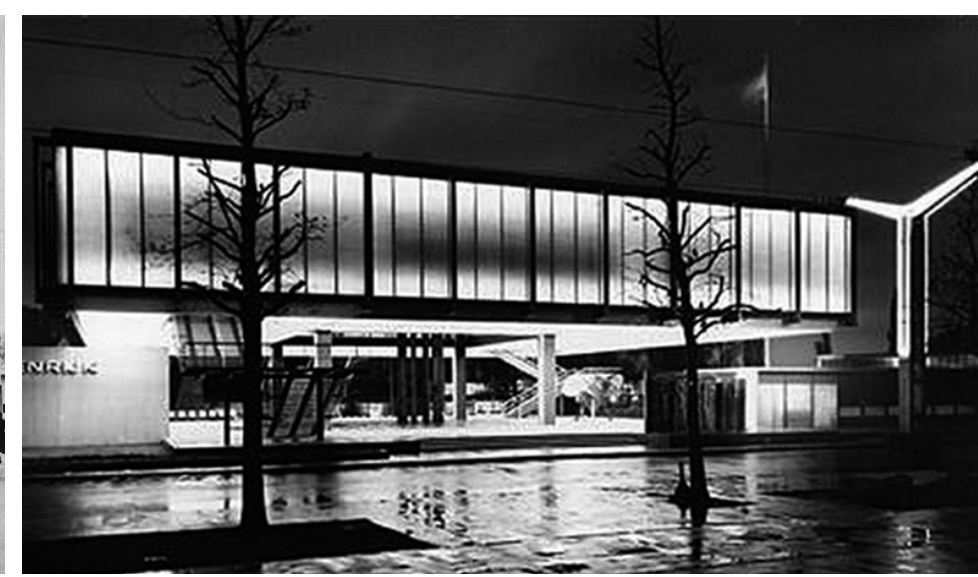

[10]
38 Oteiza y Puig no acusan a Niemeyer directamente de copia, pero realizan la siguiente comparación: "No pretendemos afirmar que Niemeyer se ha inspirado en nuestro proyecto, aunque sería interesante confrontar las fechas y examinar la lógica de la creación en ambos casos: en el nuestro, la arquitectura queda suspendida horizontalmente y se cierra por el estímulo de la estatua exterior espacialmente vacía y que le llega por la viga volada horizontalmente como elemento de integración de estatua y arquitectura. En Niemeyer, el bloque horizontal y suspendido como signo escultórico, presta su espacio interior como aprovechamiento de la arquitectura". OTEIZA, J.; PUIG, R. [Observación sobre las soluciones en el proyecto español] [escrito y fotografias, 11 hojas]. 1960. AFMJO, ID: 18054, p. 5. Disponible en: http:// www.museooteiza.org/catalogos/documentacion/detalles.php?id=18054\&bd=fondod.

39 LE CORBUSIER. "El espacio inefable". En: Minerva, junio 2006, $n^{\circ} 2$, p. 8. Disponible en: http://www.revistaminerva.com/articulo. php?id=42 [consultado 09/02/2014]. Publicado originalmente en: LE CORBUSIER. "L'espace indicible". L'Architecture d'Aujourd'hui. abril 1946, $\mathrm{n}^{\circ}$ extraordinario "Art", pp. 9-17.

${ }^{40}$ Hasta el descubrimiento del crómlech en 1957, sus obras son pensadas para generar una activación del vacio, con un espectador que como observador participa de ellas pero siempre de una manera exterior, ajena. En esta etapa final el espectador se convierte en el centro de la perspectiva, lo habita. Oteiza consigue el vacío en la estatua, pero da un paso más. La persona es el centro de ese vacío, la que lo vive, la que le da sentido. El espectado ya no está situado fuera, se encuentra dentro de la estatua. Esta es un vacio que se activa por el hombre, se convierte en un espacio receptivo para el espectador. No es un espacio fenomenológico, sino existencial, como refugio espiritual.
"Se abre entonces una profundidad sin límites que borra los muros, expulsa las presencias contingentes y realiza el milagro del espacio inefable. Ignoro el milagro de la fe, pero vivo a menudo el espacio inefable, cúspide de la emoción plástica"39.

El modo adecuado de integración de las artes y la arquitectura Oteiza lo identifica en los pabellones de Finlandia y de Austria, cuyos espacios interiores muestran un diseño tranquilo, sobrio, espacial o, empleando el término oteiziano, "receptivo"40 para el habitante. Una verdadera síntesis del arte con la arquitectura, tal como señalaba en el pabellón de Aalto en la Exposición Universal de Nueva York (1939), pues no aparecen piezas artísticas colocadas o superpuestas a ella. Se trata de arquitecturas que evitan espectáculos formales, volcadas en sí mismas, con un gran control en su relación con el exterior y con una apuesta clara por potenciar el espacio interior.

Pero Oteiza, como creador de espacios, se sirve de su habitar de lo construido y de su propia experiencia fenomenológica de la arquitectura para conformar la propuesta arquitectónica del Monumento a Batlle. Además de la referencia al cementerio de Verdún, de la que se sirve para extraer una síntesis formal que genera el monumento, las referencias formales con el pabellón de Austria tanto en la elevación de la caja contenedora del espacio como en su opacidad e intimidad, la ponen en relación con el Monumento.

Sin dejar a un lado su trabajo escultórico, el viaje de Irún hasta Bruselas forma parte del proceso creativo de Oteiza, que está intrínsecamente ligado a cuestiones arquitectónicas: las experiencias, en este caso experiencias habitadas y recuerdos de espacios vividos, se incorporan al desarrollo de la propuesta arquitectónica concreta, el Monumento a Batlle; los conceptos se trasvasan de una disciplina a otra en un proceso final, conclusivo, total, que le permitieron trabajar a todas las escalas desde la estatua al espacio urbano.

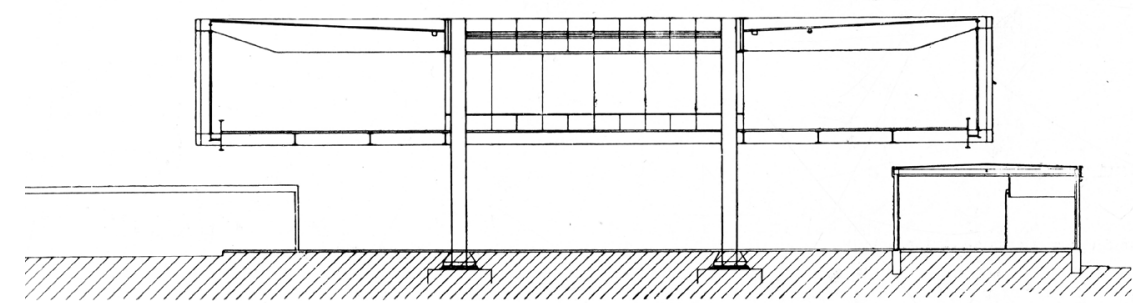

[11] [12]

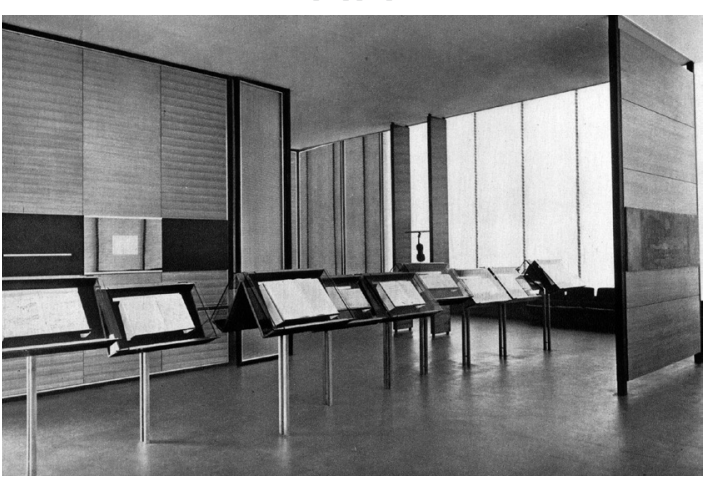

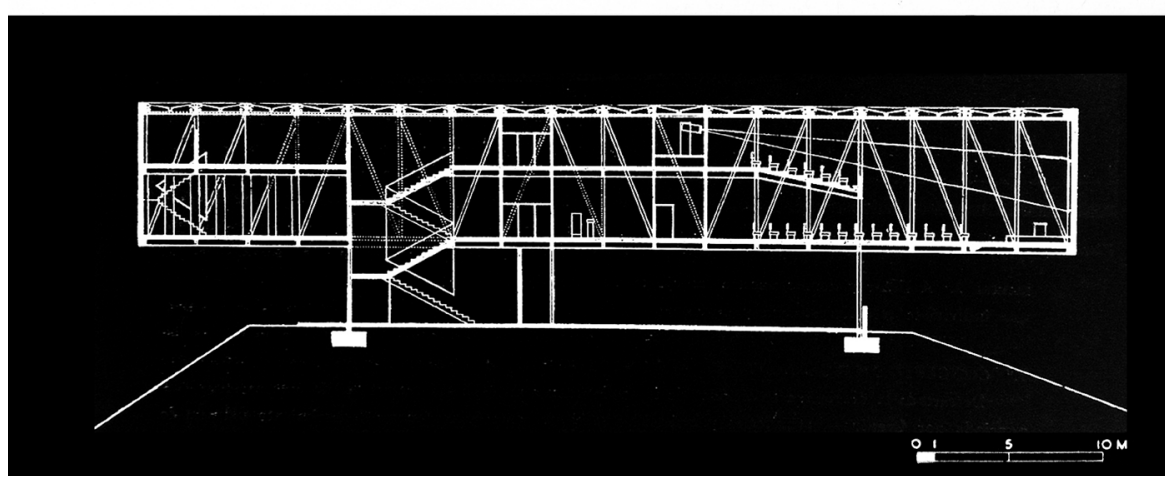

